\title{
Case Report \\ Lymphadenitis as a Rare Side Effect of H1N1 Vaccine in a Child
}

\author{
Zuhal Gundogdu and Mualla Seyhogullari \\ Kocaeli Medical Centre, 41100 Kocaeli, Turkey \\ Correspondence should be addressed to Zuhal Gundogdu, z.gundo@gmail.com
}

Received 23 August 2010; Accepted 5 November 2010

Academic Editor: Robert S. Dawe

Copyright ( 2010 Z. Gundogdu and M. Seyhogullari. This is an open access article distributed under the Creative Commons Attribution License, which permits unrestricted use, distribution, and reproduction in any medium, provided the original work is properly cited.

We present a 5-year-old boy who had the complaint of swelling and pain on the right vaccine shot and right axillary areas. The right axillary area was diagnosed as reactive lymphadenitis, which we believe is a rare local side effect of the swine flu vaccine. The key message to take away from this case is that the patient had lymphadenitis as a local side effect of the swine flu vaccine. Lymphadenitis should be reported as a possible local side effect of the swine flu vaccine.

\section{Introduction}

$2009 \mathrm{H} 1 \mathrm{~N} 1$ influenza also called Swine Flu, is caused by a new strain of the influenza virus, and it has spread through many countries. Vaccines are available to protect against 2009 H1N1 influenza a vaccine, like any medicine, could cause serious problems such as a severe allergic reaction. However, the risk of any vaccine causing serious harm, or death, is extremely small [1].

Approximately $44 \%$ of people who were administered swine flu vaccine reported mild side effects within 7 days of receiving the first dose of swine flu vaccine of CSL Biotherapies. $2.5 \%$ of the vaccine recipients reported yet moderate local side effects, and there were no severe adverse events reported following immunization [2].

Lymphadenitis is the inflammation and/or enlargement of a lymph node. The most common symptoms of lymphadenitis are swelling of one or more lymph nodes which may feel slightly hardened and may be painful when touched.

\section{The Case Study}

On December 9, 2009, a previously healthy 5-year-old boy with no history of illness was brought into the paediatrics clinic with complaints of pain in the upper part of the right arm (vaccine shot area), accompanied by swelling, bruising, and pain in the right axillary without any sign of fever.
His past medical history, family history, and social history are unremarkable.

He had swine flu vaccine administered intramuscularly in a local health authority clinic on the 8th of December 2009. Novartis H1N1 vaccine was administered to the child with a signed parental consent form requested by the Turkish Ministry of Health. Parents of the boy realised a swelling in the right arm where the vaccine was administered as well as a swelling in the right axillary area on the night of the same day when the boy started to complain about a pain. When he was brought into the paediatrics clinic on December 9, 2009, a day after the vaccination, examination revealed a hard and painful mass of nearly $2 \mathrm{~cm}$ in diameter on the right upper arm shot area with two other painful but small right axillary swellings. The examination of the other systems revealed no pathology. For the differential diagnosis, axillary ultrasonography (USG) examination was requested alongside tests for blood count (CBC) and C-reactive protein (CRP). Laboratory investigations of CBC and CRP were normal. The patient was not given any anti-inflammatory, antiallergic or antibiotic treatments.

The boy was called into the clinic three days later on the 12th of December 2009 for a physical examination; it was found that swelling of the vaccine shot area still existed although the pain had decreased. The parents turned down our request to take a biopsy for histopathological examination. 


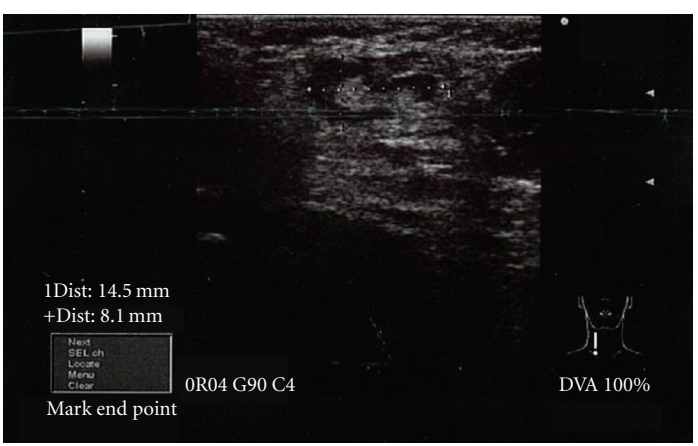

(a)

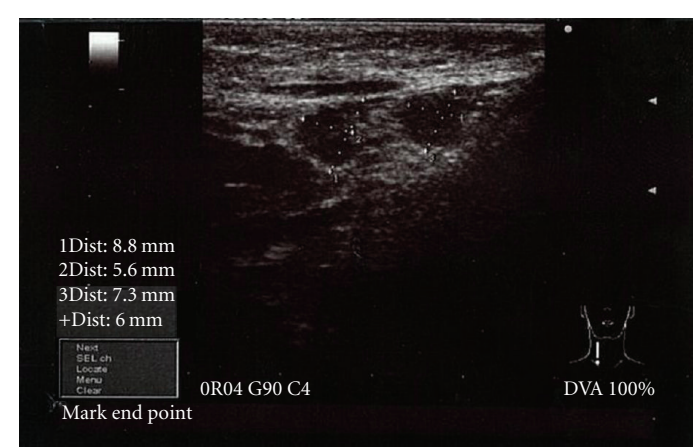

(b)

FIGURE 1: USG scans of the right axillary area taken one day after the vaccination on the 9th of December 2009.

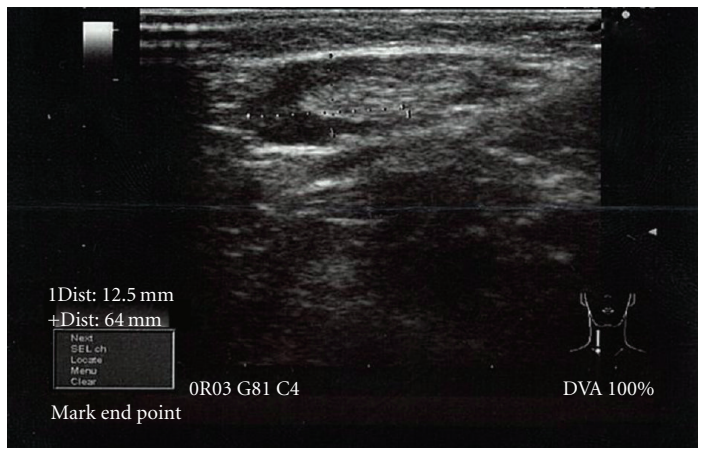

(a)

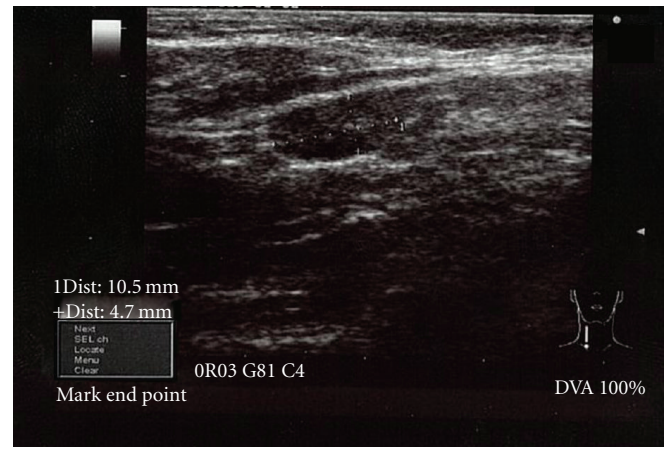

(b)

FIGURE 2: USG scans of the right axillary area taken 2 weeks after the vaccination on the 22nd of December 2009.

Ultrasound is a useful imaging modality in assessment of lymph nodes, and its features can help only in identifying abnormal nodes including size, shape, echogenic hilus, hypoechogenicity or isoechogenicity, echogeneity, coagulation necrosis, and a sharp nodal border [3].

Ultrasound features can help identify whether lymphadenitis is reactive or not. In this case, three USG results showed that this is a postvaccine lymphadenitis because of long-to-short-axis ratio (L/S ratio), oval shape, and hyperechoic hilum.

A right axillary USG showed a reactive lymph nodule in the superior area with a size of $15 \times 8 \mathrm{~mm}$ (Figure $1(\mathrm{a})$ ) and two in the inferior area with dimensions of $9 \times 6 \mathrm{~mm}$ and $7 \times 6 \mathrm{~mm}$ (Figure 1(b)). They were of fuzzy form shape with hypoechogenic cortex with clear echogenic hilus indicator. In the Doppler mode, only hilus vascular structures were observed. These findings are meaningful for acute reactive lymphadenitis. Fuzzy form and echogenic hilus indicate a benign lymphadenitis as hypoechogenic cortex is usually observed in acute cases.

The boy had been called into the outpatients' clinic a week later on the 16th of December 2009 when a second USG taken did reveal that $7 \times 6 \mathrm{~mm}$ nodule had disappeared and there were no significant changes in the structure and size of the other two nodules. Bruising had completely disappeared. However, the third USG scan carried out two weeks later on the 22nd of December 2009 revealed a decrease in nodule dimensions from $15 \times 8 \mathrm{~mm}$ to $12 \times 6 \mathrm{~mm}$ (Figure $2(\mathrm{a})$ ) and the second nodule changed from $9 \times 6$ to $10 \times 4 \mathrm{~mm}$ as shown in Figure 2(b). Furthermore, a decrease in the cortex echo and increased hilus echogeneity were also observed indicating reactive acute lymphadenitis. The physical examination carried out on the fifteenth day revealed that the patient's arm was back to normal.

\section{Discussion}

$86 \%$ of the volunteers who received Novartis's H1N1 (7.5 $\mu \mathrm{g}$ of MF59 adjuvanted) vaccine reported adverse reactions after one or both doses- the most common local side effect experienced was a pain in the injection site. The reactions were generally mild or moderate and resolved themselves after 72 hours [4]. The majority of reported local adverse events, or side effects occurring at the location where either vaccine had been administered, include tenderness pain, redness, hardening of skin, swelling, and bruising $[2,4]$. The boy presented in this report started complaining about pain and swelling on the night of the same day he had received H1N1 vaccination. 
Systemic effects were also reported by CSL Biotherapies and Novartis vaccine recipients. Approximately 36\% of volunteers who received the swine flu vaccine manufactured by CSL experienced mild systemic side effects. $8 \%$ of the vaccine recipients reported moderate systemic side effects, and less than $1 \%$ experienced a severe adverse reaction to immunization. Severe side effects reported include malaise, muscle pain, and nausea. Muscle aches were the most common systemic side effect reported by participants receiving the H1N1 vaccine produced by Novartis, and no severe systemic side effects were reported. The following are common whole-body side effects occurring in response to either H1N1 vaccination: headache, malaise, muscle pain, chills, nausea, fever, and vomiting. In addition, researchers evaluated the occurrence of select neurological adverse events $[2,4]$.

When a lymph node rapidly increases in size, its capsule stretches and causes pain. Pain is usually the result of an inflammatory process [3]. Most inflammatory diseases involve lymph nodes diffusely and homogeneously, generally preserving their oval shape. If threshold value of the longto-short-axis ratio (L/S ratio) employed is low, then the accuracy of US in differentiating normal/reactive node (oval shape) from pathogic nodes (rounded shape) is also relatively low (sensitivity $71 \%$, specifity $65 \%$ ) [5]. If the ratio used is 2.0 , sensitivity increases to $81-95 \%$ and specifity to $67-96 \%$. The second parameter to be assessed is the hyper echoic central line of lymph nodes (the hilum). The sonographic detection of the hyper echoic hilum has always been related to the probable benign nature of the lymph node [5].

Postvaccinal lymphadenitis is a reactive response of the lymph node to the vaccination. Some of the vaccines such as $B C G$, varicella zoster, and pneoumococcal can cause reactive lymphadenitis, and it is a rather common complication of BCG vaccination $[6,7]$. Local reactions (generally erythema and induration with or without tenderness) are common after the administration of vaccines containing diphtheria, tetanus, or pertussis antigens. Occasionally, a nodule may be palpable at the injection site of adsorbed products for several weeks [8].

We followed this patient for nearly twenty days but we did not encounter any systemic reaction. This was not a suppurative lymphadenitis characterized by appearance of fluctuation with erythema and edema of the overlying skin. The size of lymphadenitis has decreased and disappeared completely after 15 days.

We are of the opinion that further study is required for this type of side effect and a detailed histopathological examination could be of use. The parents turned down our request to take a biopsy for histopathological examination. However, our results have been conclusive enough that this case was a reactive lymphadenitis.

In a conclusion, reactive lymphadenitis is an unusual side effect of swine flu vaccination requiring further study, and to the best of our knowledge no cases in children have previously been reported in the literature neither by CDC nor by WHO sources, apart from a case reported in an adult [9].

\section{Acknowledgment}

Both authors would like to thank Associate Professor O. Gundogdu of Kocaeli University, Turkey, for his help.

\section{References}

[1] Vaccine Information Statement, "H1N1 Inactivated Influenza Vaccine," Department of Health and Human Services, Centers for Disease Control and Prevention, 2009.

[2] M. E. Greenberg, M. H. Lai, G. F. Hartel et al., "Response to a monovalent 2009 influenza A (H1N1) vaccine," New England Journal of Medicine, vol. 361, no. 25, pp. 2405-2413, 2009.

[3] http://www.doctorslounge.com/oncology/diagnosis/lymph_ node/axillary.htm.

[4] T. W. Clark, M. Pareek, K. Hoschler et al., "Trial of 2009 influenza A (H1N1) monovalent MF59-adjuvanted vaccine," New England Journal of Medicine, vol. 361, no. 25, pp. 2424$2435,2009$.

[5] L. Solbiati and G. Rizzatto, "Ultrasound of superficial structures high frequencies, doppler and interventional procedures," 1995.

[6] J. S. Goraya and V. S. Virdi, "Bacille Calmette-Guérin lymphadenitis," Postgraduate Medical Journal, vol. 78, no. 920, pp. 327-329, 2002.

[7] J. B. Milstien and J. J. Gibson, "Quality control of BCG vaccine by WHO: a review of factors that may influence vaccine effectiveness and safety," Bulletin of the World Health Organization, vol. 68, no. 1, pp. 93-108, 1990.

[8] "Update: Vaccine Side Effects, Adverse Reactions, Contraindications, and Precautions Recommendations of the Advisory Committee on Immunization Practices (ACIP)," http://www. cdc.gov/mmwr/preview/mmwrhtml/00046738.htm.

[9] H. Toy, D. Karasoy, and M. Keser, "Lymphadenitis caused by H1N1 vaccination: case report," Vaccine, vol. 28, no. 10, pp. 2158-2160, 2010. 


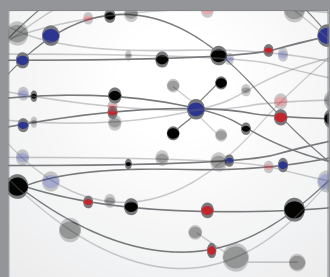

The Scientific World Journal
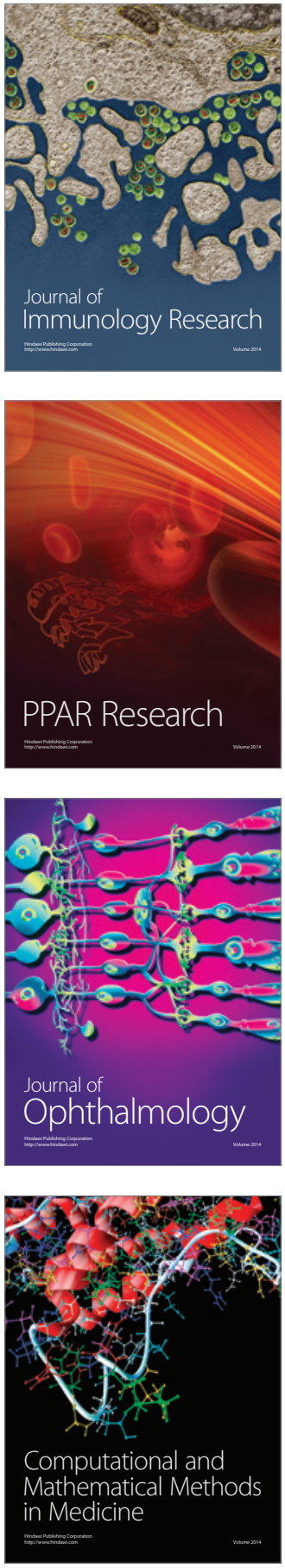

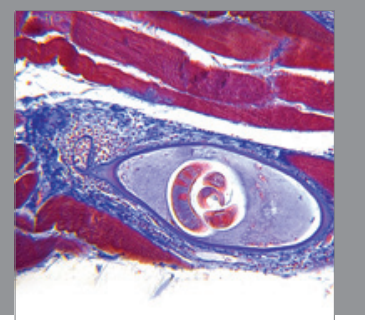

Gastroenterology

Research and Practice
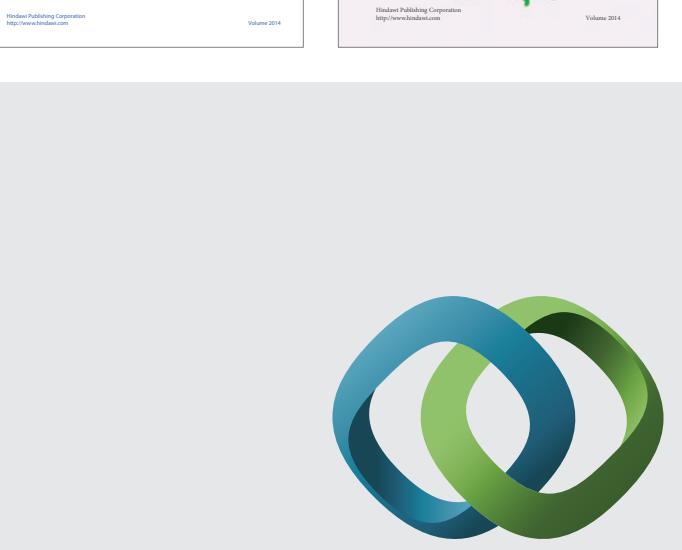

\section{Hindawi}

Submit your manuscripts at

http://www.hindawi.com
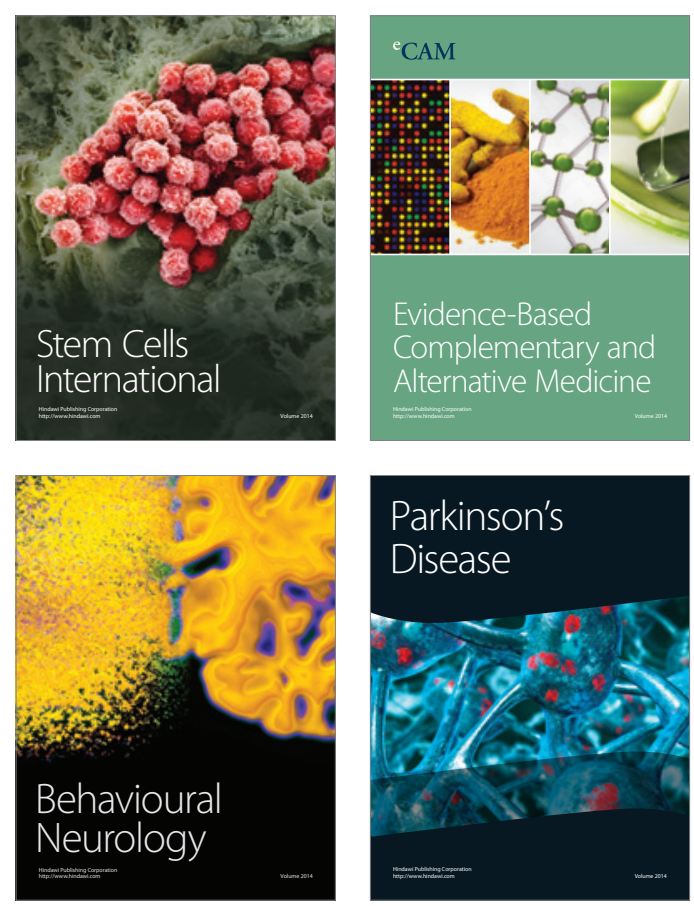

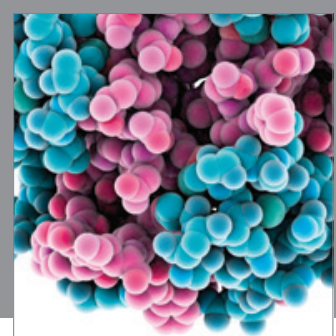

Journal of
Diabetes Research

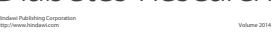

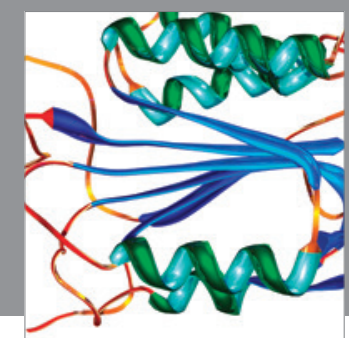

Disease Markers
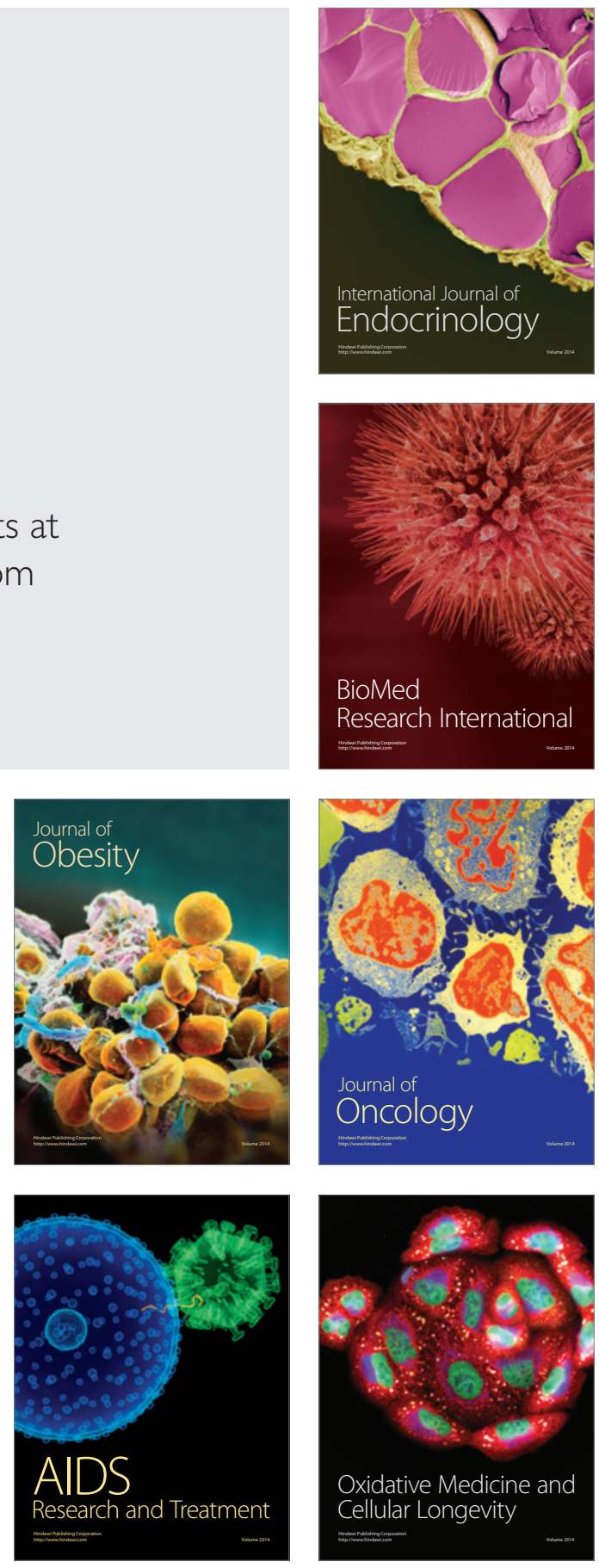